\title{
Marine biosecurity post-border management: developing incursion response systems for New Zealand
}

\author{
DEBRA M. WOTTON* \\ CHAD L. HEWITT ${ }^{\dagger}$ \\ Ministry of Fisheries \\ P.O. Box 1020 \\ Wellington, New Zealand \\ email: chad.hewitt@fish.govt.nz \\ *Present Address: School of Biological Sciences, \\ University of Canterbury, Private Bag 4800, \\ Christchurch, New Zealand.
}

\begin{abstract}
New Zealand's approach to marine biosecurity has been to integrate the pre-border, border, and post-border management actions so as to appropriately and effectively utilise resources. Prevention is clearly better than cure, consequently pre-border and border management is paramount. There is, however, recognition that quarantine style controls result in leaky borders. Early detection of new invaders, coupled with appropriate incursion response protocols and tools, increases the likelihood that a response will: (1) be effective; and (2) cause less collateral damage. Rapid response plans for predefined, high-risk species allow an adaptive and targeted approach. The selected species are also the object of targeted surveillance in high incursion risk points of first entry into the New Zealand coastal environment.
\end{abstract}

Keywords non-indigenous species; marine biosecurity; rapid response; eradication; control; invasive species

\footnotetext{
${ }^{\dagger}$ Author for correspondence. M03090; Online publication date 3 August 2004 Received 2 December 2003; accepted 27 May 2004
}

\section{INTRODUCTION}

Non-indigenous species continue to invade marine ecosystems around the world at an increasing rate (e.g., Cohen \& Carlton 1998; Thresher et al. 1999; Hewitt 2003). These invasions can adversely affect natural ecosystems, commerce, and human health (Ruiz et al. 1997). As an isolated island nation, New Zealand possesses both unique biodiversity and the opportunity to protect it. Biosecurity is the management of risks from non-indigenous species (Parliamentary Commissioner for the Environment 2000). Prevention, early detection, and rapid response are all essential components of an effective biosecurity system (Meyerson \& Reaser 2002; Hewitt et al. 2004).

Control or eradication of invasive species is technically and financially difficult (Meyerson \& Reaser 2002), particularly in the marine environment. The most effective management strategy is to reduce the risk of introductions. Consequently, preborder and border control measures are paramount (Parliamentary Commissioner for the Environment 2000; Bax et al. 2001). However, with the continued movement of craft, people, and goods, some incursions are inevitable. Post-border management systems need to be in place to prepare for and provide an appropriate response to incursions when (not if) they happen (Parliamentary Commissioner for the Environment 2000).

Early detection of new invaders increases the likelihood that a response will be effective and cause less collateral damage (see Myers et al. 2000; Bax et al. 2001). Appropriate surveillance programmes should be established that target the highest risk pathways, species, and sites. Surveillance efforts need to be coupled with incursion response systems, which will enable rapid and effective response to new introductions.

An effective response system consists of three main components: (1) processes and plans to guide response actions; (2) tools with which to respond; and (3) the capability and resources to carry out the response. A fourth component is the management of 
sustained political and public will. This paper outlines the New Zealand government's marine biosecurity incursion response processes in relation to the first component.

\section{GENERIC INCURSION RESPONSE PROTOCOL}

The Ministry of Fisheries developed a generic incursion response protocol for marine biosecurity to guide decision-making during a response. The protocol will also ensure that decisions are transparent, consistent, and well documented. This protocol is based on the Biosecurity Council Policy Statement on Responding to an Exotic Organism Incursion (Biosecurity Council 2001). The Biosecurity Act 1993 provides the legal basis for incursion response in New Zealand. The Chief Technical Officer for Marine Biosecurity (CTOMB) - a statutory position appointed under the Biosecurity Act-is responsible for managing any response.

The Ministry of Fisheries developed a decision tree outlining the key steps in the decision-making process during an incursion response (Fig. 1). Our decision tree differs somewhat from that of Bax et al. (2001), which was proposed for controlling already established invasive species, not new incursions. Long-term management has the relative luxury of time to conduct in-depth research, in contrast to incursion response where a successful outcome may hinge on a rapid response. During an incursion there may not be the time or the need for detailed research (Simberloff 2003). Although the decision tree shows activities occurring in sequence, in reality some activities will often occur simultaneously. The following section discusses the main stages of an initial response. Heading numbers correspond to steps outlined in Fig. 1.

\section{Identify species}

The first phase of a response is to confirm that a new organism (any species that has not previously been in New Zealand) has indeed been detected. The incursion response protocol is triggered when we receive notification that a suspected new organism has been found. Information on the organism is collected, including a description of the organism and the location of the infestation to enable followup if required.

Accurate identification of invasive species is fundamental to our ability to respond rapidly to new introductions (Meyerson \& Reaser 2002; Hewitt et al. 2004). The invasive northern Pacific seastar, Asterias amurensis, was misidentified in Tasmania, Australia as a native species for nearly 10 years. By the time it was correctly identified the population had exploded to pest proportions (Goggin 1998). The Ministry of Fisheries maintains contracts for rapid diagnosis of suspected new organisms. Some species may require further taxonomic investigation, although lack of taxonomic certainty does not preclude a response where it is justified. Cryptogenic species (species that are not demonstrably native or introduced; sensu Carlton 1996) are problematic for biosecurity managers, particularly in the marine environment where native biodiversity is poorly described. The status of many species remains uncertain (Hewitt 2002). Improved knowledge of New Zealand's marine biodiversity and maintenance of taxonomic capability are essential for effective biosecurity. Consequently, one of the primary outcomes of a biosecurity research strategy must be support for fundamental taxonomic work (Hewitt et al. 2004).

\section{Establish nature and magnitude of problem}

The second step is to establish the nature and magnitude of the incursion including an initial assessment of the potential risk the species poses. If an organism is confirmed as a new introduction to New Zealand, the CTO-MB will clarify whether it is already an Unwanted Organism under the Biosecurity Act. Unwanted Organisms are those species that "the Chief Technical Officer believes are capable or potentially capable of causing unwanted harm to any natural and physical resources or human health" (Biosecurity Act 1993). Unwanted Organism status enables a wide range of powers in the Biosecurity Act to be used to manage the organism. A response may still be undertaken even if a species is not determined an Unwanted Organism.

If it is not already an Unwanted Organism, the CTO-MB will undertake a preliminary risk assessment based on available information. Based on this assessment, the CTO-MB will decide whether the incursion warrants establishing a technical advisory group to provide independent advice on relevant scientific, operational, and community issues drawn from experts within New Zealand and overseas. Technical advisory groups can be established in advance of species' arrival in New Zealand, and have been used to identify likely response actions for species with known impacts. A delimitation and environmental survey may be conducted to establish 


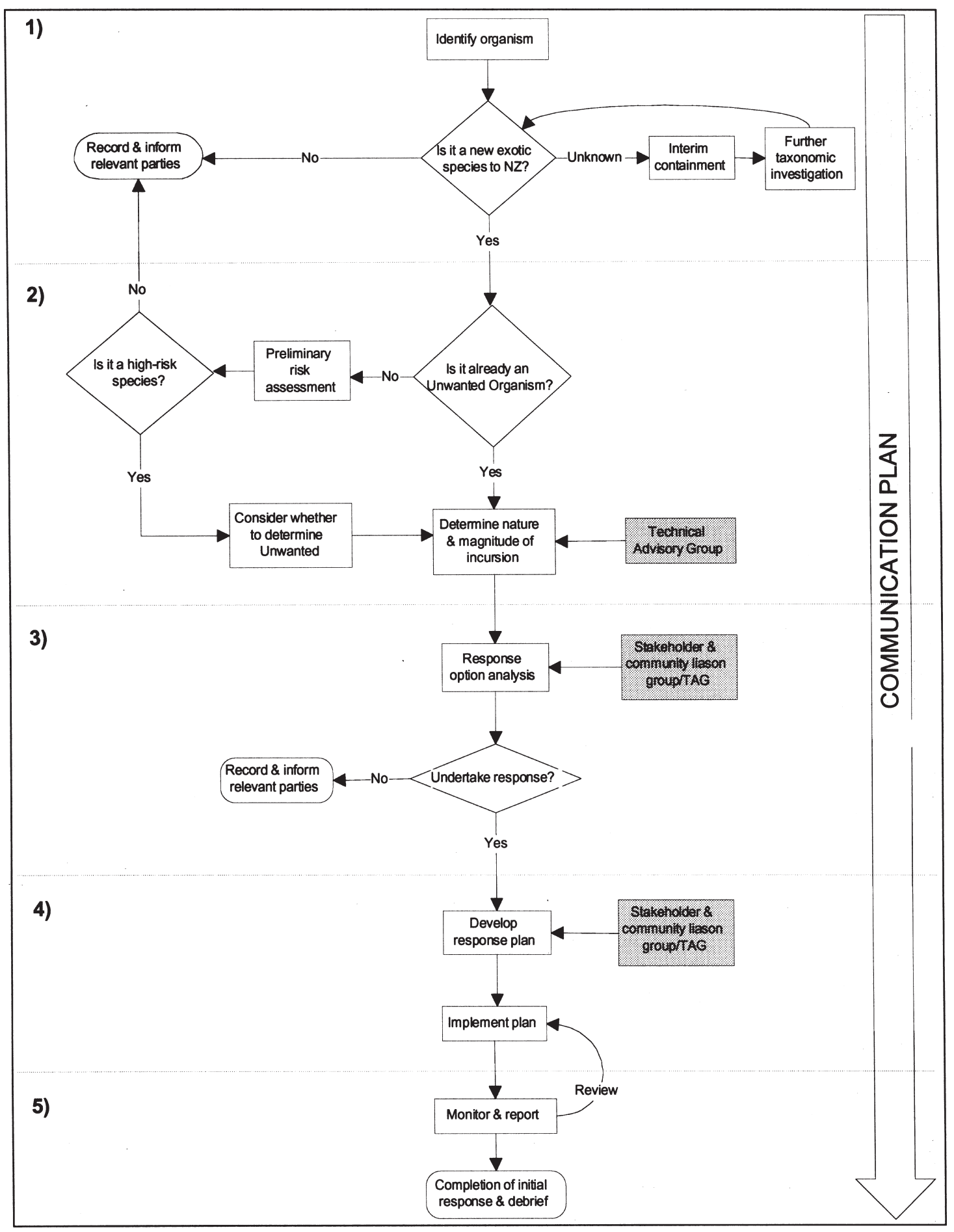

Fig. 1 Decision tree used when responding to non-indigenous organism incursions in New Zealand's marine environment. Although the diagram shows key decisions and activities occurring in sequence, in practice some of these activities may occur simultaneously. (TAG, Technical Advisory Group.) 
the distribution of the organism. A delimitation survey may not be appropriate where the organism is already known to be widespread, is benign in nature, or is unlikely to have a significant impact. The organism's potential for further spread, both by natural means and associated with human vectors, is also assessed.

A technical advisory group may contribute to a risk assessment of the organism using any additional information that has been collected in the interim. The CTO-MB may decide to determine the species an Unwanted Organism on the basis of this assessment. We have developed incursion response risk assessment guidelines based on recognised environmental risk management standards (Standards Australia/Standards New Zealand 1999, 2000). The Ministry of Fisheries Marine Biosecurity Risk Management Framework (RMF) establishes the context for this risk assessment (Cox unpubl. data).

The first step in the risk analysis is to determine the likelihood of an impact occurring if the incursion is left untreated. The likelihood analysis takes into account the potential distribution and abundance (both spatially and temporally) of the organism in New Zealand. This is based on the assumption that the more widespread an introduced species is, and the greater its abundance, the more likely it is to have adverse effects. The analysis considers the characteristics of both the organism and the recipient environment when determining the potential distribution and abundance of the introduced species in New Zealand. These include temperature and salinity tolerances, habitat requirements (e.g., depth, substrate, wave exposure), natural and human-mediated dispersal capabilities, fecundity, availability of vacant niches (e.g., paucity of taxonomic or ecological equivalents), and presence of potential natural predators, parasites, herbivores, or pathogens.
The impact analysis determines the potential effects of the introduced species on the four core values identified in the RMF (healthy environment, vibrant commerce, strong communities, and high quality recreation; Cox unpubl. data). An example of the qualitative measures of impact on a healthy environment is presented in Table 1. Each of the four values is assigned a level of impact based on the invasive potential of the species (e.g., the reproductive, dispersal, and establishment capabilities, growth rate, previous history of invasiveness, and whether closely related taxa are invasive).

In practice, it is usually difficult to determine the potential impact that a particular non-indigenous species will have. Biological invasions involve complex ecological interactions with outcomes that are often unpredictable (Ruiz et al. 1997; Ross et al. 2003). Biosecurity managers frequently have to make decisions based on incomplete biological information. To recognise this dilemma explicitly, the risk assessment process assigns scores that reflect the degree of certainty in the level of impact.

The level of risk is evaluated for each of the core values by combining the likelihood with the impact and comparing it to predetermined categories. If the CTO-MB determines that the species potentially poses an unacceptable risk, the response proceeds to the next phase.

\section{Determine appropriate response}

A response may range from providing stakeholders with information and monitoring the event through to commencing a full-scale containment, management, or eradication programme. Management options may include slowing the spread of the organism or actively managing established populations. Containment, management, or eradication responses are most likely where sustained cost-effective action

Table 1 Qualitative measures of impact on a healthy environment.

\begin{tabular}{|c|c|c|}
\hline Descriptor & Example & Level \\
\hline Catastrophic & Ecosystem collapse & 5 \\
\hline Major & $\begin{array}{l}\text { Major observable changes in ecosystem level processes, species interactions, } \\
\text { and community structure, including changes in habitat, species composition, } \\
\text { productivity, abundance, and distribution }\end{array}$ & 4 \\
\hline Moderate & $\begin{array}{l}\text { Observable changes in natural community structure and species interactions } \\
\text { although the effects are manageable with significant effort }\end{array}$ & 3 \\
\hline Minor & $\begin{array}{l}\text { Limited observable changes in natural community structure and species } \\
\text { interactions and the effects are manageable with low effort }\end{array}$ & 2 \\
\hline Insignificant & $\begin{array}{l}\text { Natural community apparently unaltered except for the addition of the } \\
\text { non-indigenous species }\end{array}$ & 1 \\
\hline
\end{tabular}


is possible, and the organism poses an unacceptable risk (as determined using the risk assessment guidelines). The actual level of response will depend on: (1) potential impacts of the organism on the environment, economy, and people; (2) technical feasibility of response options; (3) ability to target the invasive species; (4) risks associated with the treatment; (5) the degree of special public concern or interest; and (6) likelihood of the organism being eradicated or effectively managed (Biosecurity Council unpubl. policy).

In some circumstances, immediate containment or other management actions may be necessary while decisions are made on eradication or longer-term management. If the CTO-MB considers (based on the assessment of risk and available options) that further management actions are warranted, the response proceeds to the next phase. Communication of decisions to stakeholders are a final aspect of this stage.

\section{Develop and implement plan}

At this stage a response plan is developed, in consultation with stakeholders. Stakeholder input to both an assessment of response options and the development of a response plan is critical, especially when conflicting values exist. Tensions are sometimes inevitable between the need for rapid decisions and the need for adequate information (Biosecurity Council 2003) and consultation.

Response plans vary depending on both the species and the selected response option. A plan may include vector management, population control or eradication, and research. All plans contain a communication strategy to facilitate effective consultation with relevant stakeholders. The plan is implemented with appropriate monitoring and review.

\section{Monitor and review}

Monitoring and review are essential both during and after an incursion response. Response plans need to be sufficiently flexible to adapt to unexpected situations or to be reviewed and refined on the basis of new information. To ensure success, eradication programmes must monitor for any individuals missed by the treatment(s) for long enough to ensure all individuals have been eradicated.

The frequency and duration of monitoring will vary depending on the organism. For example, the Ministry of Fisheries recently completed a response to the invasive seaweed Undaria pinnatifida in which monitoring was undertaken every 30 days for 3 years. This was based on the rapid maturity of
Undaria sporophytes (after 40 days) and a maximum known gametophyte dormancy period of two years (Wotton et al. in press).

An overall review of the response is also conducted upon its completion. A substantial debrief with the response team and any advisory and liaison groups is carried out and recorded to aid in future decision-making.

The initial response is complete when one or more of the following applies: diagnosis confirms that the organism is indigenous; the organism is nonindigenous, but unwanted organism status is not warranted; a delimiting and/or environmental survey has been completed to confirm the apparent and potential distribution and abundance of the organism; no further response action is required or practicable (for example, where the organism has been eradicated, or initial actions prove ineffective and there is no practicable alternative, or no suitable response options are available); and/or sufficient arrangements are in place to sustain an appropriate level of response until decisions about a longer-term response are made (Biosecurity Council unpubl. policy).

\section{Prevent reinvasion}

Preventing reinvasion is essential to the success of any eradication programme (Myers et al. 2000). An investigation of the organism's introduction pathway into New Zealand is undertaken. Where the findings of the investigation indicate that it is appropriate, the CTO-MB can require border or other riskmanagement measures to be adjusted to mitigate the risks of repeat incursions. New Zealand's marine biosecurity border control measures tend to be broadbrush approaches that aim to reduce all accidental introductions. Species-specific refinements to these controls may not be practical. However, long-term marine bioinvasion patterns can indicate where new or improved border controls are required. We are assessing the effectiveness of our border controls by establishing baselines of what species are already here and undertaking ongoing monitoring to detect new introductions.

\section{UNWANTED ORGANISM RAPID RESPONSE PLANS}

Surveillance and response programmes need to be designed to target the greatest threats. We are developing rapid response plans for Unwanted Organisms not currently present in New Zealand. The plans will outline the operational requirements 
to respond to incursions of high-risk species. Plans will identify any available control methods, equipment required, and contain a list of relevant scientific and technical experts. They will also include information on the species' invasion history, biology, distribution, and potential vectors.

With the help of New Zealand scientists, the Ministry of Fisheries identified the following six species that pose a significant threat to New Zealand: Asterias amurensis (northern Pacific seastar), Caulerpa taxifolia, Carcinus maenas (European shore or green crab), Eriocheir sinensis (Chinese mitten crab), Potamocorbula amurensis (Asian clam), and Sabella spallanzanii (Mediterranean fanworm). The criteria used to identify these species included a history of introduction in other countries, links to a current invasion vector acting in New Zealand, suitable environmental conditions in New Zealand for the species survival, and demonstrated ecological, economic, or social impacts. The CTOMB determined these species Unwanted Organisms under the Biosecurity Act in 2000. They were also included in the Biosecurity Act Notifiable Organisms Order 2002. This list of threat species was initially developed for a public surveillance network and will be refined as more information becomes available.

Incursion response preparation is closely linked to surveillance. The Ministry of Fisheries has established a marine biosecurity surveillance programme using a risk-based approach. Surveillance efforts are targeted at the highest risk pathways, species, and areas. We are undertaking active surveillance for the above six Unwanted Organisms, initially focusing on eight harbours with a high risk of incursion.

\section{CONCLUSIONS}

Although responding to non-indigenous species incursions is difficult in the marine environment, a response can still be effective with appropriate planning and tools. The Ministry of Fisheries has established a transparent incursion response process that is consistent with other biosecurity agencies and the Biosecurity Council Policy (Biosecurity Council unpubl. policy). Recognising that marine biosecurity is limited in both capacity and capability, the emphasis must be on early detection and the development of tools for rapid identification and response.

\section{ACKNOWLEDGMENTS}

We thank R. Allibone and L. Chadderton for the opportunity to participate in the Invasive Species: Fish \& Fisheries Workshop during the Australian Society of Fish Biology annual conference in Wellington, 30 June-1 July, 2003. Thanks also to significant contributions by Ministry of Fisheries staff, especially Chris O'Brien, Maria Cassidy, Camilla Cox, and Neville Smith. Geoff Hicks and Rachel Garthwaite commented on the protocol and risk assessment guidelines. Thanks to Chris O'Brien for commenting on a draft of this manuscript. The Cawthron Institute, NIWA, and Te Papa helped MFish to identify species that pose a threat to New Zealand.

\section{REFERENCES}

Bax, N.; Carlton, J. T.; Mathews-Amos, A.; Haedrich, R. L.; Howarth, F. G.; Purcell, J. E.; Rieser, A.; Gray, A. 2001: The control of biological invasions in the world's oceans. Conservation Biology 15(5): 1234-1246.

Biosecurity Council 2003: Tiakina Aotearoa Protect New Zealand: The Biosecurity Strategy for New Zealand. Wellington, Biosecurity Council. 63 p.

Carlton, J. T. 1996: Biological invasions and cryptogenic species. Ecology 77(6): 1653-1655.

Cohen, A. N.; Carlton, J. T. 1998: Accelerating invasion rate in a highly invaded estuary. Science 279: 555-558.

Goggin, C. L. ed. 1998: Proceedings of a meeting on the biology and management of the introduced seastar Asterias amurensis in Australian waters. Technical Report 15. Hobart, Australia, Centre for Research on Introduced Marine Pests, CSIRO.

Hewitt, C. L. 2002: The distribution and diversity of tropical Australian marine bio-invasions. Pacific Science 56: 213-222.

Hewitt, C. L. 2003: Marine biosecurity issues in the world oceans: global activities and Australian directions. Ocean Yearbook 17: 193-212.

Hewitt, C. L.; Willing, J.; Bauckham, A.; Cassidy, A. M.; Cox, C. M. S.; Jones, L.; Wotton, D. M. 2004: New Zealand marine biosecurity: delivering outcomes in a fluid environment. New Zealand Journal of Marine and Freshwater Research 38: 429-438.

Meyerson, L. A.; Reaser, J. K. 2002: Biosecurity: moving toward a comprehensive approach. BioScience 52(7): 593-600.

Myers, J. H.; Simberloff, D.; Kuris, A. M.; Carey, J. R. 2000: Eradication revisited: dealing with exotic species. Trends in Ecology and Evolution 15(8): 316-320. 
Parliamentary Commissioner for the Environment 2000: New Zealand under siege: a review of the management of biosecurity risks to the environment. Wellington, New Zealand, Office of the Parliamentary Commissioner for the Environment.

Ross, D. J.; Johnson, C. R.; Hewitt, C. L. 2003: Variability in the impact of an introduced predator (Asterias amurensis: Asteroidea) on soft sediment assemblages. Journal of Experimental Marine Biology and Ecology 288: 257-278.

Ruiz, G. M.; Carlton, J. T.; Grosholz, E. D.; Hines, A. H. 1997: Global invasions of marine and estuarine habitats by non-indigenous species: mechanisms, extent, and consequences. American Zoologist 37: 621-632.

Simberloff, D. 2003: How much information on population biology is needed to manage introduced species? Conservation Biology 17(1): 83-92.
Standards Australia/Standards New Zealand 1999: Risk management AS/NZS 4360. Sydney, Standards Australia, and Wellington, Standards New Zealand.

Standards Australia/Standards New Zealand 2000: Environmental risk management-principles and process. Sydney, Standards Australia, and Wellington, Standards New Zealand.

Thresher, R. E.; Hewitt, C. H.; Campbell, M. L. 1999: Synthesis: introduced and cryptogenic species in Port Phillip Bay. In: Hewitt, C. L.; Campbell, M. L.; Thresher, R. E.; Martin, R. B. ed. Marine biological invasions of Port Phillip Bay, Victoria. entre for Research on Introduced Marine Pests Technical Report No. 20. Hobart, CSIRO Marine Research. Pp. 283-295.

Wotton, D. M.; O’Brien, C.; Stuart, M.; Fergus, D. J. in press: Eradication success down under: Heat treatment of a sunken trawler to kill the invasive seaweed Undaria pinnatifida. Marine Pollution Bulletin.

\section{ADDENDUM}

As part of the Biosecurity Strategy Implementation, the Ministry of Agriculture and Forestry has been identified as the lead agency for biosecurity delivery in central government, and marine biosecurity functions have been transferred from the Ministry of Fisheries. 
